\title{
PERFIL EPIDEMIOLÓGICO DE GESTANTES PORTADORAS DE HIVIAIDS NO BRASIL
}

\author{
EPIDEMIOLOGICAL PROFILE OF PREGNANT WOMEN WITH \\ HIVIAIDS IN BRAZIL
}

Cleisla Tamires Lacerda Silva ${ }^{1}$
Karla Pereira Vasconcelos
Hirisdiane Bezerra Alves

RESUMO: Objetivo: descrever o perfil epidemiológico de gestantes portadoras de HIV/Aids no Brasil. Metodologia: trata-se de uma revisão na literatura referente às gestantes portadoras de HIV/Aids. O levantamento bibliográfico foi realizado no mês de dezembro de 2019, utilizando as bases de dados Lilacs, Medline, SciELO e Google Acadêmico. Foram incluídos trabalhos completos, em português, publicados entre 2015 a 2019. Apósavaliação, foram identificados 10 artigos que preencheram todos os critérios de inclusão e exclusão. Resultados: a maioria das gestantes tinham entre os 20 a 35 anos de idade, ensino médio incompleto, eram brancas, donas de casa, tiveram o diagnóstico de HIV antes da gestação, fazem pré-natal e uso de terapia antirretroviral. Conclusão: a implantação de ações de prevenção na gestação, parto e puerpério são as formas mais eficientes para reduzir o risco de transmissão vertical. Assim, podemos observar a importância de conhecer o perfil das gestantes portadoras de HIV/Aids, para que o governo e serviços de saúde tenham ações voltadas para essa população de acordo com oseu contexto social.

Palavras chave: HIV. Síndrome da Imunodeficiência Adquirida. Gravidez. Epidemiologia. Perfil de Saúde.

ABSTRACT: Objective: to describe the epidemiological profile of pregnant women with HIV / AIDS in Brazil. Methodology: this is a review of the literature regarding pregnant women with HIV / AIDS. The bibliographic survey was conducted in December 2019, using the Lilacs, Medline, SciELO and Google Scholar databases.

\footnotetext{
${ }^{1}$ Graduanda do Curso de Enfermagem da Universidade Estadual da Paraíba - UEPB.

2 Graduanda do Curso de Enfermagem da Universidade Estadual da Paraíba - UEPB, karlapv2017@gmail.com.

${ }^{3}$ Enfermeira pelo Centro Universitário Maurício de Nassau/ Campina Grande. Pós-graduanda em Urgência, Emergência e UTI pelo Instituto Michele Sales/ Campina Grande. Pós-graduanda em Obstetrícia e Neonatologia pelo Instituto Michele Sales/ Campina Grande. dianyalves06@gmail.com.
} 
Complete works, in Portuguese, published between 2015 and 2019 were included. After evaluation, 10 articles were identified that met all inclusion and exclusion criteria. Results: most pregnant women were between 20 and 35 years of age, incomplete high school, white, housewives, diagnosed with HIV before pregnancy, undergoing prenatal care and using antiretroviral therapy. Conclusion: the implementation of preventive actions during pregnancy, childbirth and the puerperium are the most efficient ways to reduce the risk of vertical transmission. Thus, we can observe the importance of knowing the profile of pregnant women with HIV / AIDS, so that the government and health services have actions aimed at this population according to their social context.

Keywords: HIV. Acquired Immunodeficiency Syndrome. Pregnancy. Epidemiology. Health Profile. 


\section{INTRODUÇÃO}

A Aids é um dos principais problemas de saúde na atualidade, devido ser uma infecção grave e global. Os infectados pelo vírus da imunodeficiência humana (HIV) têm sua função imunológica comprometida. O vírus pode ser transmitido através de contato sexual, pelo sangue, incluída neste a transmissão vertical, e por meio da amamentação (BRASIL, 2010).

Com o passar das décadas, o perfil epidemiológico da transmissão do HIV passoupor modificações. Houve aumento de sua contaminação através de relações heterossexuais, que pode ter ocorrido devido o aumento de casos no sexo feminino, inclusive em mulheres em idade fértil (SILVA et al. 2013). No Brasil, foram notificadas 125.144 gestantes portadoras de HIV no período de 2000 a 2019 (Brasil, 2019).

A transmissão vertical pode ocorrer durante o período gestacional, no periparto, que compreende o trabalho de parto e nascimento, ou no pós-parto por meio da amamentação (KAKEHASI et al, 2014). Por volta de 35\% das transmissões acontecem no período intraútero, especialmente no fim da gestação, $65 \%$ das infecções ocorrem no periparto, e 7 a 22\% através da amamentação (BRASIL, 2012).

Diversos aspectos influenciam no risco de transmissão vertical do HIV. Desta forma, tem-se feito o estudo de várias intervenções utilizadas durante a gestação para reduzir a taxa de transmissão materno-fetal. Identificar as características sociais e o estado de saúde maternos podem apontar as intervenções adequadas para prevenir a transmissão.Deve-se começar pela identificação da infecção materna pela testagem no pré-natal e momento do parto (KAKEHASI et al, 2014).

No Brasil, casos de infecção de HIV e a AIDS fazem parte da Lista Nacional de Notificação Compulsória de Doenças, estando a AIDS como notificação compulsória desde 1986 e o HIV desde 2014 (BRASIL, 2019). O Programa Nacional de DST e Aids tem como uma de suas prioridades diminuir as taxas de transmissão 
vertical no país. Entre as ações implantadas por protocolos de tratamento que têm mostrados bons resultados estão o uso de antirretrovirais para profilaxia e de alimentação por meio de fórmulas pelos bebês, até a confirmação de sua situação sorológica (BRASIL, 2010).

É fundamental que todas as gestantes façam testes para detectar o HIV, que é realizado no $1^{\circ}$ e $3^{\circ}$ trimestre durante o pré-natal e no momento do parto. As grávidas com diagnóstico positivo devem iniciar o tratamento com uso de antirretrovirais para prevenir a transmissão vertical e tratar de sua saúde (BRASIL, 2017). No Brasil, com o início da terapia antirretroviral (TARV) em 1996, os portadores tiveram aumento de sobrevida e de sua qualidade de vida (BRASIL, 2010).

Sem intervenções, há uma taxa de transmissão vertical de cerca de 25,5\%, e estudos mostram que com intervenções para prevenção essa taxa reduz para 1 a $2 \%$, entre elas estão o uso de antirretrovirais combinados, a realização de parto cesáreo eletivo, o uso de zidovudina profilática na parturiente e no recém-nascido, e a não amamentação (BRASIL, 2012).

Para entender o processo de adoecimento, é necessário observar todas as circunstâncias que contribuem para o mesmo, como os fatores culturais, sociais, econômicos, políticos, gênero, etnia, entre outros (SOUZA; MIRANDA; FRANCO, 2011).

Mesmos com os avanços referentes à qualidade de vida, prognóstico, tratamento e redução da mortalidade observados nas últimas décadas, a Aids permanece como uma doença grave e incurável, que necessita de ações sociais e do governo com objetivando sua prevenção (SILVA et al. 2013).

Devido essas circunstâncias, é de fundamental importância a realização da vigilância epidemiológica e de estudos sobre a mesma para o controle da transmissão vertical, pelo ato que a vigilância epidemiológica avalia a dimensão do problema e a realidade local por um período, conseguindo um retrato epidemiológico a partir do qual serão realizadas medidas de prevenção e planejamento de propostas de controle da infecção (LIMA et al, 2014).

Devido esse contexto, o objetivo desse trabalho foi descrever o perfil epidemiológico de gestantes portadoras de HIVIAids no Brasil. 


\section{METODOLOGIA}

Trata-se de uma revisão na literatura, que teve como questão norteadora: Qual o perfil epidemiológico das gestantes portadoras de HIV/Aids no Brasil? O levantamento bibliográfico foi realizado no mês de dezembro de 2019, por meio de busca on-line de produções científicas nacionais sobre o perfil de gestantes soropositivas. A obtenção das publicações ocorreu através de buscas processadas nas seguintes bases de dados:Literatura Latino-americana e do Caribe em Ciências da Saúde (Lilacs), Medical Literature Analysis and Retrieval System Online (Medline), Scientific Eletronic Library Online (SciELO) e Google Acadêmico. Foram utilizandos como descritores para as buscas: "gestantes", "HIV", "Aids", e "perfil epidemiológico".

Utilizou-se como critérios de inclusão trabalhos completos, com publicação em português entre 2015 e 2019, e que abordassem o perfil epidemiológico de gestantes portadoras de HIV/aids no Brasil. Foram excluídos trabalhos de revisão, anais de congresso, teses, relatos de experiência, e os que não responderam aos critérios de inclusão.

Foram identificadas 138 publicações, das quais foi realizada a leitura dos resumos dos artigos e excluídos aqueles que não atendiam ao objetivo do estudo. Após avaliação, foram excluídas 128 publicações e identificados 10 artigos que preencheram todos os critérios de inclusão estabelecidos. Com a leitura dos artigos, foi elaborada uma tabela (Tabela 1), contendo as seguintes informações: ano de publicação, autores, título e objetivo. Posteriormente à análise, foram extraídas informações acerca do perfil da gestantes soropositivas, observando a predominância de faixas etárias, ocupacão, escolaridade, estado civil, raça/cor, realização de pré-natal, momento de diagnóstico, usode terapia antirretroviral, uso de álcool, tabaco e drogas, e presença de coinfecções, além dos estados e regiões em que foram feitas as pesquisas dos artigos. 


\section{RESULTADOS}

Os dez artigos selecionados foram publicados entre 2015 e 2019, mostrando comoa produção científica sobre HIV/Aids em gestantes é um assunto importante no contexto atual da sociedade brasileira. Os artigos são mostrados na tabela 1, que mostra os anos depublicação, autores, título e objetivo.

Tabela 1 - Autores, títulos, anos de publicação e objetivos dos artigos selecionados.

\begin{tabular}{|c|c|c|c|}
\hline Autores & Título & Ano & Objetivo \\
\hline $\begin{array}{l}\text { Araújo, E. C. et } \\
\text { al. }\end{array}$ & $\begin{array}{lr}\text { Avaliação } & \text { da } \\
\text { abordagem rem } & \text { médica } \\
\text { em } & \text { gestantes hiv } \\
\text { positivas } & \end{array}$ & 2015 & $\begin{array}{l}\text { Avaliar a conduta médica em } \\
\text { relação à mulher HIV positiva, } \\
\text { durante a gestação, o trabalho de } \\
\text { parto e o parto. }\end{array}$ \\
\hline $\begin{array}{l}\text { Ayala, A. L. M.; } \\
\text { Moreira, A.; } \\
\text { Francelino, G. }\end{array}$ & $\begin{array}{l}\text { Características } \\
\text { socioeconômicas } \\
\text { fatores associados à } \\
\text { positividade para o hiv } \\
\text { em gestantes de } \\
\text { uma cidade do sul do } \\
\text { Brasil }\end{array}$ & 2016 & $\begin{array}{l}\text { Conhecer as características } \\
\text { socioeconômicas e as relacionadas } \\
\text { à positividade de gestantes para } \\
\text { HIV. }\end{array}$ \\
\hline Bick, M. A. et al. & 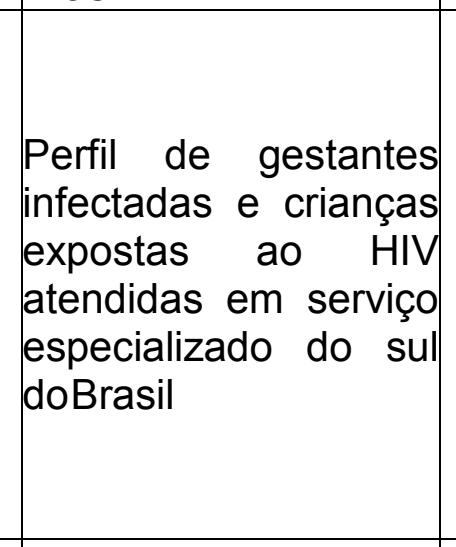 & 2018 & $\begin{array}{l}\text { Caracterizar o perfil social e clínico } \\
\text { de gestantes infectadas pelo HIV e } \\
\text { os fatores associados à prevenção } \\
\text { da transmissão vertical, bem como } \\
\text { analisar a qualidade das } \\
\text { informações disponíveis nas fichas } \\
\text { de notificação do SINAN e } \\
\text { prontuários clínicos de gestantes } \\
\text { infectadas e crianças expostas ao } \\
\text { HIV, em um serviço especializado, } \\
\text { no interior do Rio Grande do Sul. }\end{array}$ \\
\hline $\begin{array}{l}\text { Brandão, M. N.et } \\
\text { al. }\end{array}$ & $\begin{array}{l}\text { Desafios } \\
\text { prevenção } \\
\text { transmissão verticaldo } \\
\text { HIV em Petrolina }- \text { PE } \\
\text { e Juazeiro-BA }\end{array}$ & 2016 & $\begin{array}{l}\text { Aferir taxa de transmissão vertical } \\
\text { (TTV) do HIV e descrever a adoção } \\
\text { das medidas profiláticas (MP) em } \\
\text { gestantes infectadas e crianças } \\
\text { expostas, nos municípios de } \\
\text { Petrolina/PE e Juazeiro/BA. }\end{array}$ \\
\hline
\end{tabular}




\begin{tabular}{|c|c|c|c|}
\hline $\begin{array}{l}\text { Lima, S. K. S. S. } \\
\text { et al. }\end{array}$ & $\begin{array}{ll}\text { Caracterização } & \text { das } \\
\text { gestantes } & \text { com } \\
\text { hiv/aids admitidas em } \\
\text { hospital de referência }\end{array}$ & 2017 & $\begin{array}{l}\text { Descreve as características clínico } \\
\text { epidemiológicas das Gestantes } \\
\text { infectadas com HIVIAIDS. }\end{array}$ \\
\hline $\begin{array}{l}\text { Maia, M. M. M. } \\
\text { et al. }\end{array}$ & \begin{tabular}{|lr} 
Prevalência & de \\
infecções & congênitas \\
e perinatais & em \\
gestantes & HIV \\
positivas da região \\
metropolitana de \\
$\begin{array}{ll}\text { Horizonte } \\
\text { Holo }\end{array}$ \\
\end{tabular} & 2015 & $\begin{array}{l}\text { Avaliar a prevalência de } \\
\text { toxoplasmose, rubéola, } \\
\text { citomegalovirose, hepatites B e C e } \\
\text { sífilis (Torchs) em uma coorte de } \\
\text { gestantes, bem como identificar os } \\
\text { fatores sociodemográficos, clínicos } \\
\text { e laboratoriais. }\end{array}$ \\
\hline $\begin{array}{l}\text { Paes, A. L. V. et } \\
\text { al. }\end{array}$ & 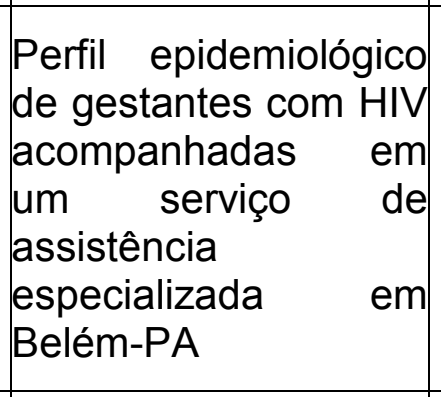 & 2017 & $\begin{array}{lll}\text { Identificar o perfil epidemiológico } \\
\text { das gestantes com HIV } \\
\text { acompanhadas pelo Serviço de } \\
\text { Atendimento Especializado do } \\
\text { Hospital Universitário João de } \\
\text { Barros Barreto (HUJBB), em } \\
\text { Belém- PA, no período de janeiro } \\
\text { de } 2008 \text { a dezembro de } 2017 \text {. } \\
\end{array}$ \\
\hline Silva, C. M. et al. & \begin{tabular}{|llr} 
Panorama & & \\
epidemiológico & do \\
HIV/aids & & em \\
gestantes & de & um \\
estado do & Nordeste \\
brasileiro & &
\end{tabular} & 2018 & $\begin{array}{l}\text { Conhecer as características } \\
\text { epidemiológicas da infecção pelo } \\
\text { HIV em gestantes. }\end{array}$ \\
\hline Silva, L. R. et al. & $\begin{array}{l}\text { Variáveis } \\
\text { epidemiológicas da } \\
\text { infecção pelo HIV em } \\
\text { gestantes }\end{array}$ & 2016 & $\begin{array}{l}\text { Traçar o perfil epidemiológico das } \\
\text { gestantes com vírus da } \\
\text { imunodeficiência humana (HIV) } \\
\text { atendidas em uma maternidade de } \\
\text { referência estadual no município de } \\
\text { Teresina-PI. }\end{array}$ \\
\hline Sousa, S. F. et al. & $\begin{array}{l}\text { Perfil de gestantes } \\
\text { comhivassistidas na } \\
\text { policlínica Degurupi- } \\
\text { TO }\end{array}$ & 2015 & $\begin{array}{l}\text { Realizar uma análise dos } \\
\text { prontuários de gestantes com HIV } \\
\text { registrados na Policlínica. }\end{array}$ \\
\hline
\end{tabular}

Os estudo foram realizados em 10 estados brasileiros, onde 05 (Alagoas, Pernambuco, Bahia, Rio Grande do Norte e Piauí) foram realizados na região Nordeste, 02 (Pará e Tocantins) na região Norte, 02 (Rio rande do Sul e Santa Catarina) no Sul e 01 (Minas Gerais) no Sudeste. O estudo de Brandão et al. (2016) foi realizado em dois estados (Pernambuco e Bahia), e os estudos de Araújo et al. (2015) e Paes et al. (2017) foram ambos realizados no Pará.

Em todos os estudos, a faixa etária predominante se encontrava entre os 20 a 35 anos de idade, período no qual as mulheres se encontram em plena idade 
reprodutiva. Noestudo de Araújo et al. (2015), as adolescentes (menores de 19 anos) representaram $18,6 \%$ da amostra, dado preocupante, pois mostra que as adolescentes têm uma vida sexual ativa que algumas vezes ocorre de forma desprotegida.

Quanto à ocupação, Bick et al. (2018) observaram que a maioria $(59,4 \%)$ foram identificadas como do lar/donas de casa, o que também foi observado nos estudos de Lima, S. K. S. S. et al. (2017), Ayala, Moreira e Francelino (2016) e Silva et al. (2016).

Com relação à escolaridade, a maioria dos estudos relataram predominância do ensino fundamental incompleto (BICK et al., 2018; BRANDÃO et al., 2016; LIMA, S.

K. S. S. et al., 2017; MAIA et al., 2015; PAES et al., 2017; SILVA et al., 2016; SILVA et al., 2018). No estudo de Ayala, Moreira e Francelino (2016), a maioria (31\%) das gestantes possuíam ensino médio completo, assim como nos estudos de Araújo et al. (2015) e Sousa et al. (2015).

Quanto ao estado civil, no estudo de Lima, S. K. S. S. et al. (2017) prevaleceramas solteiras $(42,3 \%)$, seguido pelas mulheres em união estável $(26,9 \%)$ e as casadas (17,3\%). Já no de Silva et al. (2016) prevaleceu a união estável, e no de Maia et al. (2015)a união estável/casadas.

Cinco estudos observaram a variável cor/raça. No estudo de Lima, S. K. S. S. et al. (2017) houveram $46,1 \%$ de mulheres brancas, predominância também observada no estudo de Ayala, Moreira e Francelino (2016) e no de Sousa et al. (2015). Diferente destes estudos, Silva et al. (2018) relataram que $72,1 \%$ das gestantes se autodeclararam pardas, sendo que a cor branca representou apenas $11,8 \%$, e também o estudo de Brandão et al. (2016) possuía $88,2 \%$ das gestantes não brancas.

No estudo de Brandão et al. (2016) foi descrito que 97,1\% das gestantes relataramter recebido atendimento de pré-natal, predominância essa encontrada em outros estudos (ARAÚJO et al., 2015; AYALA; MOREIRA; FRANCELINO, 2016; SILVA et al. 2018;SILVA et al., 2016).

No estudo de Bick et al. (2018), 60,1\% das gestantes tiveram o diagnóstico antesda realização do pré-natal, assim como nos estudos de Brandão et al. (2016) e 
Maia et al. (2015). Porém, nos estudos de Silva et al. (2018), Paes et al (2017) e Silva et al. (2016), as gestantes que tiveram diagnóstico de HIV durante o pré-natal liderou na maioria dos casos.

Sousa et al. (2015) retratam que 83,3\% das gestantes fizeram uso de terapia antirretroviral para prevenção da transmissão vertical do HIV, e a terapia também foi utilizada pela maioria das gestantes em outros cinco estudos que analisaram a variável (AYALA; MOREIRA; FRANCELINO, 2016; BICK et al., 2018; BRANDÃO et al., 2016; MAIA et al., 2015; SILVA et al., 2016). Araújo et al. (2015) destacam que a zidovudina (AZT) profilática foi iniciada no segundo trimestre de gestação em $52,8 \%$ daspacientes.

Araújo et al. (2015) observaram em seu estudo que $11,4 \%$ das gestantes faziam uso de drogas ilícitas. Já no estudo de Paes et al. (2017) foi relatado que $18,08 \%$ eram etilistas, $15,95 \%$ eram etilistas e tabagistas, $14,43 \%$ eram apenas tabagistas e 14,88\% faziam apenas uso de drogas ilícitas. No estudo de Lima, S. K. S. S. et al. (2017), 9,6\% eram etilistas, 9,6\% tabagistas e 5,7 \% faziam uso de drogas ilícitas.

No que se refere à coinfecções, foi relatado por Bick et al. (2018) a presença de Hepatite C (4,5\%), Papiloma Vírus Humano (2,7\%), Sífilis $(18,2 \%)$ e Tuberculose $(0,9 \%)$. No estudo de Maia et al. (2015), 9,9\% das gestantes tinham alguma sorologia positiva para toxoplasmose, rubéola, citomegalovirose, hepatites B e C e/ou sífilis.

No estudo de Paes et al. (2017), foi citada a presença de algumas infecções oportunistas presentes, com destaque da candidíase oral em 34,04\% dos casos, e as diarreias crônicas infecciosas em $22,35 \%$.

\section{DISCUSSÃo}

Os resultados estão de acordo com o Boletim Epidemiológico HIV/Aids de 2019 (BRASIL, 2019), em que este relata a predominância de gestantes jovens, na faixa etária de 20 a 24 anos, com escolaridade da $5^{\circ}$ a $8^{\circ}$ série incompleta, porém 
com aumento de mulheres com nível médio completo. Houve divergência apenas quanto à raça/cor, pois, diferente deste estudo, a prevalência é de mulheres pardas, seguido de brancas. Entretanto, a maior prevalência de uma etnia em gestantes infectadas com HIV pode estar relacionada às características locais da região de cada estudo.

Neste estudo, foi observado que maioria dos artigos relataram a prevalência da faixa etária de 20 a 35 anos nas gestantes portadoras de HIV. Essa informação mostra como o processo de feminização do HIV pode levar a infecção de mulheres em plena idade reprodutiva (LIMA et al, 2014), e consequentemente a um risco de transmissão vertical. Entretanto, ao encontrar a maioria (76\%) das gestantes com HIV com idades de 25 a 39 anos, Ayala, Moreira e Francelino (2016) afirmam que esta é um resultado esperado, visto que nessa faixa etária é o período em que há maior reprodução.

Também foi relatada a presença de gestantes soropositivas adolescentes. Campos et al. (2014) relatam que pode ser identificada uma relação entre a escolaridade dos adolescentes e vulnerabilidade, de forma que a baixa escolaridade dos adolescentes interfere sobre seu poder tomada de decisão em situações de risco.

Foi possível observar que a maioria das gestantes não possuíam vínculo empregatício, realizando atividades domésticas. Esse resultado pode estar relacionado com a baixa escolaridade dessas mulheres, o que prejudica sua introdução no mercado detrabalho (AYALA; MOREIRA; FRANCELINO, 2016).

A baixa escolaridade foi relatada em todos os estudos, sendo o ensino fundamental incompleto destacado um sete estudos, e o fundamental completo predominante nos outros três. Esses dados demonstram que as mulheres mais suscetíveis à contaminação com o HIV têm uma baixa escolaridade. Passos et al. (2013) destacam que a baixa escolaridade da mulher, informação relevante pois pode proporcionar uma maior atençãoda equipe de saúde à paciente, ainda é pouco valorizada e de difícil acessso no serviço hospitalar. Além disso, o menor nível de escolaridade da mulher dificulta a busca por informações à respeito de sua saúde e dos procedimentos disponíveis. 
Silva et al. (2013) enfatizam que o fato da AIDS infectar cada vez mais pessoas desfavorecidas economicamente e de baixa escolaridade reforça a necessidade de desfazer conceitos errôneos através da educação e conscientização.

Outro quesito a ser considerado é o estado civil das gestantes, que eram em sua maioria casadas/em união estável em dois estudos, e solteiras seguido de união estável em outro. Durante a gestação, a mulher passa por várias alterações fisiológicas, que podem levar à mudanças psicológicas e emocionais e, desta forma, a presença de um parceiro pode ser de grande importância para a gestante, pois este pode estimular a mulher a ficar mais segura perante suas emoções e preocupaçoes. Deve ser observada a possibilidade dessas mulheres transmitirem o vírus para outras pessoas, devendo ser levado em conta a sua consciência de comunicar o parceiro sobre a presença da infecçãoe utilizar meios de prevenir a contaminacão (LIMA S. K. S. S. et al., 2017; SOUZA et al., 2013).

A realização de pré-natal foi demonstrada em boa parte dos trabalhos, corroborando com um amplo estudo de Domingues et al. (2015), que evidenciou que em todas as regiões do país há quase uma totalidade de cobertura pré-natal com pelo menos uma consulta no Brasil.

A presença de atendimento de pré-natal é uma informação bastante relevante, porque muitas mulheres não comparecem ao serviço de saúde com frequência, e a consulta de pré-natal acaba sendo uma das únicas oportunidades de diagnosticar o HIV eassim prevenir a transmissão vertical (LIMA, S. K. S. S. et al., 2014).

Porém, mesmo com essa alta adesão das gestantes ao pré-natal, é necessário observar o percentual de mulheres que não realizaram o pré-natal, pois esta ausência podeestar relacionada à falta de conhecimento sobre sua condição, e também demonstra uma falha do sistema de saúde na busca ativa por estas mulheres (SILVA et al., 2018).

Mesmo com a existência de diversos protocolos que orientem sobre medidas profiláticas para transmissão vertical do HIV, ainda ocorrem dificuldads para alcançar algumas gestantes no pré-natal e estimular que as mesmas sigam o tratamento. Nesse sentido, é importante identificar o perfil dessas gestantes, de forma a delinear 
ações efetivas para prevenção da transmissão vertical (BARBOSA; MARQUES; GUIMARÃES, 2018).

Em relação ao diagnóstico de HIV, em três estudos prevaleceu o diagnóstico antes do pré-natal, e três estudos tiveram a maioria das gestantes com diagnóstico durante a realização do pré-natal. Essas descobertas do diagnóstico no pré-natal demonstram a importância de realização de rastreio de HIVIAids no pré-natal, para que, assim, medidasde profilaxia sejam adotadas o mais precocemente possível.

O estudo de Domingues et al. (2015) observaram a cobertura de testagem de HIVdurante o pré-natal no Brasil, constatando que há uma alta adesão à realização do primeiro teste sorológico para o vírus (>80\%), porém no que se refere à segunda testagem, essa adesão é baixa (<30\%), demonstrando as dificuldades de executar recomendações para realização da segunda testagem para o vírus.

A taxa de detecção de HIV em gestantes no Brasil teve um aumento de 38,1\% no período de 2008 e 2018, em grande parte devido a ampliação de diagnósticos no pré-natal, resultando na prevenção da transmissão vertical do vírus. Esse aumento foi observado em todas as regiões, com exceção da Sudeste, onde houve pouca variação (BRASIL, 2019).

Lima, S. S. et al. (2017) destacam que a realização de teste para HIV em gestantese o aconselhamento para aquelas que forem soropositivas é essencial e fazem parte da assistência pré-natal. Porém, para os autores, os profissionais de saúde não são preparados ou capacitados para oferecer este aconselhamento. Destacam ainda que os cuidados às gestantes soropositivas demandam uma assistência específica da equipe de saúde, queobjetiva o controle da carga viral e, assim, a diminuição do risco de transmissão vertical.

Nos estudos foi demonstrada uma boa adesão ao uso da terapia antirretroviral. O uso de antirretrovirais é um dos principais fatores para prevenir a transmissão vertical, e devem ser utilizados durante a gestação, parto, e na criança exposta. A implantação de medidas profiláticas foi responsável pela diminuição progressiva de casos de Aids por transmissão vertical ao longo dos anos (LIMA, S. K. S. S. et al, 2014).

Nos artigos estudados, foi observado que um minoria das gestantes declararam fazer uso de álcool, tabaco, ou drogas injetáveis. Esta é uma importante 
informação, que mostra características dos hábitos dessas mulheres. É necessário enfatizar o número destas gestantes em uso de drogas, pois gestantes em uso de substâncias psicoativas são mais propensas à apresentar deficiência no autocuidado, descuido em sua própria saúde, e assim dificulta a realização de um tratamento adequado que previne a transmissão vertical, além de poder expandir a cadeia de transmissão do vírus com o uso de drogas injetáveis (BARBOSA; MARQUES; GUIMARÃES, 2018).

Entretanto, o uso de drogas pelas gestantes, sejam elas lícitas ou ilícitas, pode ser uma informação subdiagnosticada, visto que estas podem negar ou amenizar o relato do consumo devido a existência de um "sentimento de culpa" e o medo de advertência do profissional de saúde. Além disso, gestantes que são dependentes químicas têm uma adesão inferior ao pré-natal e à grupos de gestantes, e possuem maiores riscos de instercorrências obstétricas e fetais, e algumas abandonam os filhos ou são consideradas legalmente incapazes de cuidarem dos filhos. Desta forma, o uso de drogas por gestantes deve ser considerado um problema social e de saúde (KASSADA et al., 2013).

No decorrer da gestação, deve ser proporcionada uma atenção integral à mulher, observando o seu contexto social, condição socioeconômica, história de vida, e os comportamentos violentos ou autodestrutivos que possam ser gerados pelo uso de drogas ilícitas. A gravidez incentiva as mulheres em procurar o serviço de saúde, e os profissionais devem aproveitar a oportunidade para indagar sobre o uso de drogas, e assimtomar providências para melhorar a qualidade do cuidado na gestação e diminuir a morbidade materna e neonatal (MELO et al., 2014).

Em alguns estudos foi relatada a presença de coinfecções, o que evidencia a necessidade de rastreio precoce de diagnóstico de IST's nas gestantes, de forma a buscar impedir a ocorrência de complicações tanto obstétricas quanto neonatais (TRAVASSOS et al., 2013).

Dentre os vários fatores relacionados à transmissão da mãe para o bebê, destacam- se: a carga viral aumentada, a ruptura prolongada das membranas amnióticas, presença de coinfecções com ISTs, tipo de parto, a prematuridade, uso de drogas e aleitamento materno, sendo os dois primeiros os principais (BRASIL, 2012). 
Também é importante destacar os relatos de Lima S. K. S. S. et al. (2017), Silva et al. (2018) e Silva et al. (2016), sobre as informações que são deixadas em branco nas fichas das gestantes, o que demonstra falhas na coleta de dados dos serviços. Esse subregistro das informações acaba fazendo com que não haja um resultado fidedigno da real situação epidemiológica das gestantes soropositivas nas regiões estudadas, afetandoa qualidade da avaliação desses estudos.

O conhecimento de informações socioeconômicas e relacionas a infecção de HIV em gestantes é um importante recurso para a elaboração de políticas públicas voltadas para a saúde das mesmas (AYALA; MOREIRA; FRANCELINO, 2016).

\section{CONCLUSÃO}

No estudo foi observado que no perfil das gestantes portadoras de HIV/Aids, há um maior percentual de mulheres jovens, entre 20 a 35 anos, com baixo nível de instrução, que possuem união estável, brancas, donas de casa, que tiveram diagnóstico de HIV antes da gestação, fazem pré-natal e uso de terapia antirretroviral. Algumas receberam o diagnóstico de HIV durante o pré-natal, mostrando a importância de testagem para a infecção durante essas consultas, podendo ser a única oportunidade de identificação do vírus.

A implantação de ações de prevenção na gestação, parto e puerpério são as formas mais eficientes para reduzir o risco de transmissão vertical. Assim, podemos observar a importância de conhecer o perfil das gestantes portadoras de HIV/Aids, para que o governo e serviços de saúde tenham ações voltadas para essa população de acordo com oseu contexto social. 


\section{REFERÊNCIAS BIBLIOGRÁFICAS}

ARAÚJO, E. C. et al. Avaliação da abordagem médica em gestantes HIV positivas. Revista Paraense de Medicina, v. 29, n. 2, 2015.

AYALA, A. L. M.; MOREIRA, A. M.; FRANCELINO, G. Características socioeconômicas e fatores associados à positividade para o HIV em gestantes de uma cidade do sul do Brasil. Revista de APS, v. 19, n. 2, p. 210-20, 2016.

BARBOSA, B. L. F. A.; MARQUES, A. K.; GUIMARÃES. J. V. Gestantes HIV positivas e os fatores de risco relacionados à transmissão vertical do HIV. Revista de Enfermagem UFPE on line, Recife, v. 12, n. 1, p. 171-8, 2018.

BICK, M. A. et al. Perfil de gestantes infectadas e crianças expostas ao HIV atendidas em serviço especializado do Sul do Brasil. Revista Brasileira de Saúde Materno Infantil, Recife, v. 18, n. 4 , p. $803-13,2018$.

BRANDÃO, M. N. et al. Desafios na prevenção da transmissão vertical do HIV em Petrolina-PE e Juazeiro-BA. Revista Brasileira de Saúde Materno Infantil, Recife, v. 16, n. 3, p. 325-36, 2016.

BRASIL. Ministério da Saúde. Secretaria de Vigilância em Saúde. Cuidado integral àspessoas que vivem com HIV pela Atenção Básica: manual para a equipe multiprofissional. Brasília: Ministério da Saúde, 2017.

Ministério da Saúde. Secretaria de Atenção à Saúde. Gestação de alto risco:manual técnico. 5. ed., Brasília: Ministério da Saúde, 2012.

Ministério da Saúde. Secretaria de Vigilânciaem Saúde. Boletim Epidemiológico HIVAids. Brasília: Ministério da Saúde, 2019.

. Ministério da Saúde. Secretaria de Vigilância em Saúde. Doenças infecciosas e parasitárias: guia de bolso. 8. ed., Brasília: Ministério da Saúde, 2010.

CAMPOS, C. G. A. P. et al. A vulnerabilidade ao HIV em adolescentes: estudo retrospectivo em um Centro de Testagem e Aconselhamento. Revista Mineira de Enfermagem, v. 18, n. 2, p. 310-14, 2014.

DOMINGUES R. M. S. M. Et al. Prenatal testing and prevalence of HIV infection during pregnancy: data from the "Birth in Brazil" study, a national hospital-based study. BMC Infectious Diseases, v. 15, n.100, 2015.

KAKEHASI, F. M. et al. Vírus da imunodeficiência humana adquirida/HIV no período neonatal. Revista Médica de Minas Gerais, v. 24, n. 2, p. 241-247, 2014.

KASSADA, D. S. et al. Prevalência do uso de drogas de abuso por gestantes. Acta Paulista de Enfermagem, v. 26, n. 5, p. 467-71, 2013.

LIMA, A. C. M. A. C. C. et al. Avaliação epidemiológica da prevenção da transmissão vertical do HIV. Acta Paulista de Enfermagem, v. 27, n. 4, p. 311-8, 2014.

LIMA, S. K. S. S. et al. Caracterização das gestantes com HIV/Aids admitidas em hospital de referência. SANARE, Sobral, v.16, n.01, p. 45-51, 2017. 
LIMA, S. S. et al. HIV na gestação: pré-natal, parto e puerpério. Ciência \& Saúde, v. 10,n. 1, p. 56-61, 2017.

MAIA, M. M. M. et al. Prevalência de infecções congênitas e perinatais em gestantes HIV positivas da região metropolitana de Belo Horizonte. Revista Brasileira de Ginecologia e Obstetrícia, v. 37, n. 9, p. 421-7, 2015.

MELO, V. H. et al. Uso de drogas ilícitas por gestantes infectadas pelo HIV. Revista Brasileira de Ginecologia e Obstetrícia, v. 36, n.12, 2014.

PAES, A. L. V. et al. Perfil epidemiológico de gestantes com HIV acompanhadas em umserviço de assistência especializada em Belém-PA. Revista Internacional Interdisciplinar, v. 10, n. 3, p. 100-09, 2017.

PASSOS, S. C. S. et al. Aconselhamento sobre o teste rápido anti-HIV em parturientes. Revista Brasileira de Epidemiologia, São Paulo, v.16 n.2, 2013.

SILVA, C. M. et al. Panorama epidemiológico do HIV/aids em gestantes de um estado do Nordeste brasileiro. Revista Brasileira de Enfermagem, v. 71, p. 613-21, 2018.

SILVA, L. R. et al. Variáveis epidemiológicas da infecção pelo HIV em gestantes. Revista de Enfermagem da UFPI, v. 5, n. 1, p. 34-39, 2016.

SILVA, R. A. R. et al. A epidemia da AIDS no Brasil: análise do perfil atual. Revista de Enfermagem UFPE On line, Recife, v. 7, n. 10, p. 6039-8, 2013.

SOUZA, N. A. et al. Perfil epidemiológico das gestantes atendidas na consulta de prénatalde uma unidade básica de saúde em São Luís-MA. Revista de Ciências da Saúde, São Luís, v. 15, n. 1, p. 28-38, 2013.

SOUZA, S. F. et al. Perfil de gestantes com HIV assistidas na policlínica Degurupi-TO. Revista Cereus, v.7, n. 3, 2015.

SOUZA, P. K. R.; MIRANDA, K. C. L; FRANCO, A. C. Vulnerabilidade: análise do conceito na prática clínica do enfermeiro em ambulatório de HIVIAIDS. Revista Brasileira de Enfermagem, Brasília, v. 64 n. 2, 2011.

TRAVASSOS, A. G. A. et al. Prevalence of sexually transmitted infections among HIV-infected women in Brazil. The Brazilian Journal of Infectious Diseases, v. 16, n. 6, p. 581-85, 2012. 